\title{
First Test of Lorentz Invariance in the Weak Decay of Polarized Nuclei
}

\author{
S.E. Müller ${ }^{*}$ E.A. Dijck, H. Bekker J.E. van den Berg, O. Böll, \\ S. Hoekstra, K. Jungmann, C. Meinema, J.P. Noordmans, M. Nuñez Portela, \\ C.J.G. Onderwater, C. Pijpker, A.P.P. van der Poel, B. Santra, A. Sytema, \\ R.G.E. Timmermans, O.O. Versolato ${ }^{\dagger}$ L. Willmann, H.W. Wilschut $\ddagger^{\dagger}$ and K. Yai \\ Kernfysisch Versneller Instituut, University of Groningen, \\ Zernikelaan 25, 9747 AA Groningen, The Netherlands
}

(Dated: July 31, 2013)

\begin{abstract}
A new test of Lorentz invariance in the weak interactions has been made by searching for variations in the decay rate of spin-polarized ${ }^{20} \mathrm{Na}$ nuclei. This test is unique to Gamow-Teller transitions, as was shown in the framework of a recently developed theory that assumes a Lorentz symmetry breaking background field of tensor nature. The nuclear spins were polarized in the up and down direction, putting a limit on the amplitude of sidereal variations of the form $\left|\left(\Gamma_{\text {up }}-\Gamma_{\text {down }}\right)\right| /\left(\Gamma_{\text {up }}+\Gamma_{\text {down }}\right)<3 \times 10^{-3}$. This measurement shows a possible route toward a more detailed testing of Lorentz symmetry in weak interactions.
\end{abstract}

PACS numbers: 11.30.Cp, 24.80.+y, 23.40.Bw 
Lorentz invariance means that physical laws are independent of boosts and rotations. It is at the basis of all known interactions. In the weak sector relatively few tests of Lorentz invariance have been made, even though the understanding of the weak interactions has been crucial in developing the standard model. In this work we consider a new test that exploits the spin degrees of freedom in $\beta$ decay, searching for a dependence of the nuclear lifetime on the orientation of the nucleus. Recent theoretical work [1] enables relating the present test to other possible Lorentz symmetry tests in the weak interactions and put them in the overall framework developed by Kostelecký and coworkers [2]. The CPT tests of neutral-meson [3] or neutrino [4] oscillations and the $\beta$-decay endpoint spectrum [5] are also in the weak domain, however, they differ in nature.

We write the relative variation in the $\beta$-decay rate $\Gamma$ as

$$
\frac{d \Gamma}{\Gamma_{0}}=1+\vec{\beta} \cdot\left[A \frac{\langle\vec{I}\rangle}{I}+\xi_{1} \hat{n}_{1}\right]+\xi_{2} \frac{\langle\vec{I}\rangle}{I} \cdot \hat{n}_{2} .
$$

Here, $\Gamma_{0}$ is the standard model decay rate, with $\vec{\beta}$ the velocity vector of the $\beta$ particle in units of the speed of light. The nuclear polarization of the parent nucleus is $\langle\vec{I}\rangle / I$. $A$ is the $\beta$-asymmetry parameter in the standard model that violates parity. Other parameters in the $\beta$ decay of spin-polarized nuclei [1] are not relevant for this work.

Lorentz invariance violation (LIV) appears in Eq. (1) with magnitudes $\xi_{1}, \xi_{2}$ and directions $\hat{n}_{1}, \hat{n}_{2}$ relative to the emission direction of the $\beta$ particle and the polarization of the parent nucleus, respectively. The directions $\hat{n}_{i}$ need not be identical. In the theory of Ref. [1] $\xi_{i}$ and $\hat{n}_{i}$ depend on the nature of the transition, e.g. an allowed Fermi or GamowTeller transition, or whether a transition is forbidden [6]. For the current discussion Eq. (1) suffices. An overview of current experimental and theoretical work is given in Ref. [7].

Early experimental work looked for a limit on the $\beta$-emission anisotropy of unpolarized nuclei in forbidden $\beta$ decays of ${ }^{90} \mathrm{Y},{ }^{137} \mathrm{Cs}$, and ${ }^{99} \mathrm{Tc}$ [8, 9], i.e. $\xi_{1} \hat{n}_{1}$. In contrast, this work concerns $\xi_{2} \hat{n}_{2}$. A reevaluation of the earlier work is given in Ref. [6] relating it to LIV in allowed decays [1]. Within this theory the limits found from Refs. [8, 9] and our work are complementary: they probe different combinations of parameters characterizing the LIV interaction.

In the present experiment, we test Lorentz invariance by looking for a change in the decay rate of allowed $\beta$ decay of ${ }^{20} \mathrm{Na}$ when reversing the orientation of the nuclear spin $\vec{I}$, thus putting a limit on the parameter $\xi_{2} \hat{n}_{2}$. Choosing the polarization direction (denoted by $\hat{z}$ ) 


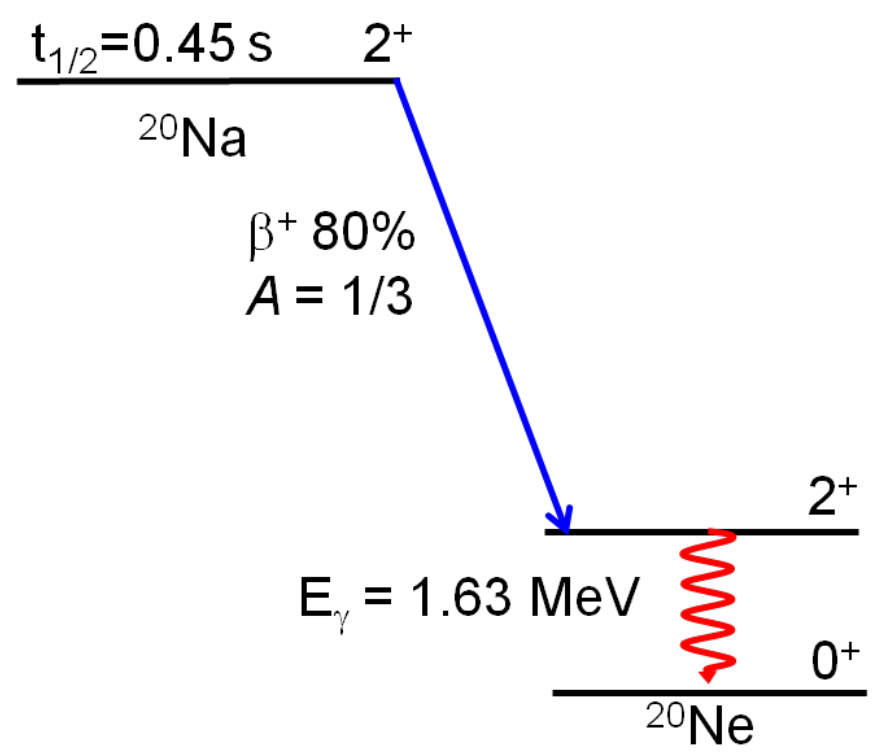

FIG. 1: Relevant part of the decay scheme of ${ }^{20} \mathrm{Na}$.

to be perpendicular to the horizontal plane, a fixed preferred direction $\hat{N}$ with components $\left\{N^{1}, N^{2}, N^{3}\right\}$ in the Sun-centered frame [10] will lead to an observation in the laboratory frame as a time dependence of the form

$$
\hat{z} \cdot \hat{n}_{2}(t)=N^{1} \sin \theta \cos \omega_{\oplus} t+N^{2} \sin \theta \sin \omega_{\oplus} t+N^{3} \cos \theta .
$$

This has two time-dependent terms with sidereal frequency $\omega_{\oplus}$ and one independent of time, with the relative amplitudes determined by the colatitude of the experiment, $\theta$.

The relevant properties [11] of ${ }^{20} \mathrm{Na}$ decay are shown in Fig. 1. ${ }^{20} \mathrm{Na}$ is a short-lived isotope $\left(t_{1 / 2}=0.45 \mathrm{~s}\right)$, which decays mostly $(80 \%)$ via $\beta^{+}$emission in a $2^{+} \rightarrow 2^{+}$GamowTeller transition, for which $A=1 / 3$. The endpoint energy for this transition is $11.2 \mathrm{MeV}$. By measuring the $\beta$ asymmetry we determine the magnitude of the polarization $\langle\vec{I}\rangle / I$. The decay of the daughter nucleus is observed from the subsequent $1.6 \mathrm{MeV} \gamma$ emission, which can be well discriminated from positron annihilation radiation at $0.511 \mathrm{MeV}$. Using the parity-even $\gamma$ decay, instead of the parity-odd $\beta$ decay, one can measure the lifetime of the parent independent of the polarization. Any residual dependence on polarization, in particular a sidereal dependence, is a measure of LIV. In our analysis we assume that the electromagnetic and strong interactions obey Lorentz symmetry.

${ }^{20} \mathrm{Na}$ is produced in the ${ }^{20} \mathrm{Ne}(\mathrm{p}, \mathrm{n}){ }^{20} \mathrm{Na}$ reaction by colliding a ${ }^{20} \mathrm{Ne}$ beam of 23 $\mathrm{MeV} /$ nucleon with hydrogen in a liquid-nitrogen-cooled gas target. The resulting isotopes 


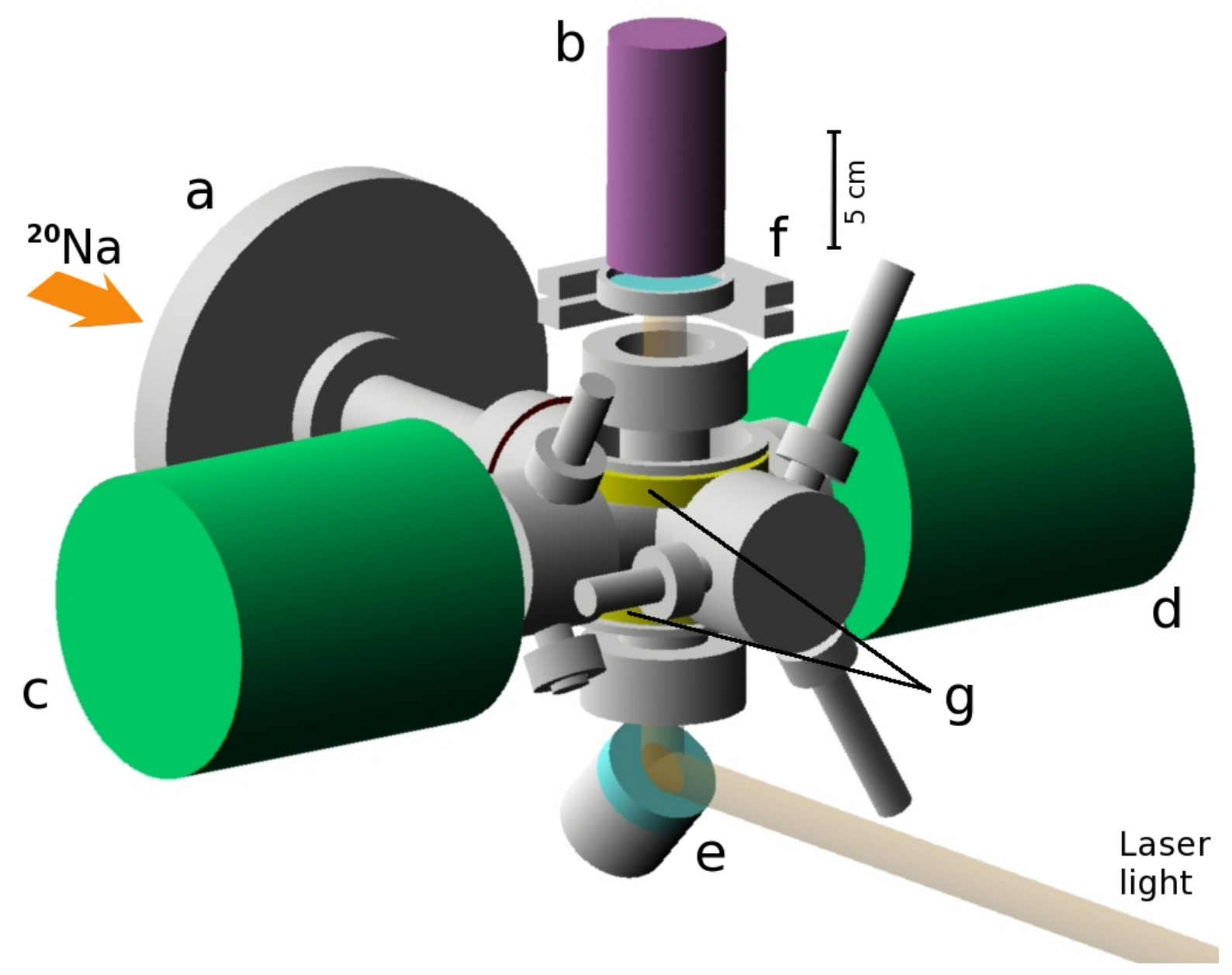

FIG. 2: Schematic drawing of the experimental setup. Shown are the flange with entrance window (a), the $\beta$ detector (b), the NaI $\gamma$ detectors (c,d), optical mirrors (e,f), and coils for the magnetic holding field $(\mathrm{g})$.

pass through the TRI $\mu \mathrm{P}$ isotope separator facility to obtain a clean ${ }^{20} \mathrm{Na}$ beam [12]. The energetic particles are stopped in a cell with Ne buffer gas at 2 atm. With adjustable aluminum degrader foils in the beam line the beam's stopping distribution is centered in the cell by maximizing the $\beta$ count rate.

The Na atoms are polarized by optical pumping. Therefore, the neon buffer gas is cleaned with a liquid-nitrogen-filled cryotrap and a gas purifier cartridge. A heatable dispenser with natural sodium is mounted inside the buffer-gas cell. The natural sodium binds residual chemically active contaminants in the gas and prevents the radioactive sodium from forming molecules. 
The stopped ${ }^{20} \mathrm{Na}$ atoms in the center of the gas cell (see Fig. 2) are optically pumped into a "stretched" state in which the electronic and nuclear spins are both aligned vertically by a magnetic holding field of about $2 \mathrm{mT}$ provided by two coils. A circularly polarized laser beam with $589 \mathrm{~nm}$ wavelength is sent through the buffer gas cell. The pumping can be achieved both via the ${ }^{2} \mathrm{~S}_{1 / 2}-{ }^{2} \mathrm{P}_{1 / 2}\left(\mathrm{D}_{1}\right)$ and the ${ }^{2} \mathrm{~S}_{1 / 2}-{ }^{2} \mathrm{P}_{3 / 2}\left(\mathrm{D}_{2}\right)$ transitions. In our pressure domain it was found from simulations that this leads to polarizations with opposite sign of about $95 \%\left(D_{1}\right)$ and $-75 \%\left(D_{2}\right)$ [13]. The latter transition has been used in this experiment. Beam blockers are employed to switch the helicity of the laser beam going through the cell. The nuclear polarization $P$ is verified by detecting $\beta^{+}$particles emitted in the upward direction with a plastic scintillator. The $\beta$ detector is set to trigger on minimum-ionizing particles. The contribution of $\gamma$ rays to its count rate can be neglected for the polarization measurement. The $\gamma$-decay rate is measured with two NaI detectors, 5 inches in diameter. The detector threshold is set above the annihilation peak at 0.511 $\mathrm{MeV}$ to a level of about $1 \mathrm{MeV}$ where the $\gamma$ spectrum is relatively flat. The $\gamma$ detectors are positioned perpendicular to the polarization direction to symmetrize the setup with respect to the $\beta$ particles. The $\beta$ - and $\gamma$-ray asymmetry are obtained by measuring the count rates in dead-time-free scalers. The scalers are read every $1 \mathrm{~ms}$. A fraction of the analog data is digitized for data-quality control.

The $\beta$ asymmetry is obtained as

$$
\mathcal{A}_{\beta}=P K_{A} \approx \frac{R_{\beta}^{\uparrow}-R_{\beta}^{\downarrow}}{R_{\beta}^{\uparrow}+R_{\beta}^{\downarrow}},
$$

where due to the high $\beta$ energies and the placement of the $\beta$ detector the analyzing power $K_{A} \approx A=1 / 3$ and $R_{\beta}^{\uparrow(\downarrow)}$ is the count rate for up(down) polarization.

The cyclotron was operated with a cycle of 1 s "on" - 1 s "off," and 0.4 s to change polarization. Data were recorded in sequences of three "on"- "off" cycles: two with opposite laser light helicity and one in which no laser light entered the buffer-gas cell. In this last period, no polarization of nuclei is expected. Figure 3 a shows the rates measured by the $\beta$ detector for different helicities of the laser light. Figure $3 \mathrm{~b}$ shows the corresponding $\beta$ asymmetry, $\mathcal{A}_{\beta}$, defined in Eq. (3). Once the beam is on, $\mathcal{A}_{\beta}$ quickly rises to a plateau of approximately 10\%, implying 30\% polarization. If all atoms are in the laser field a polarization of about $75 \%$ is expected. The observed polarization is lower because of diffusion 

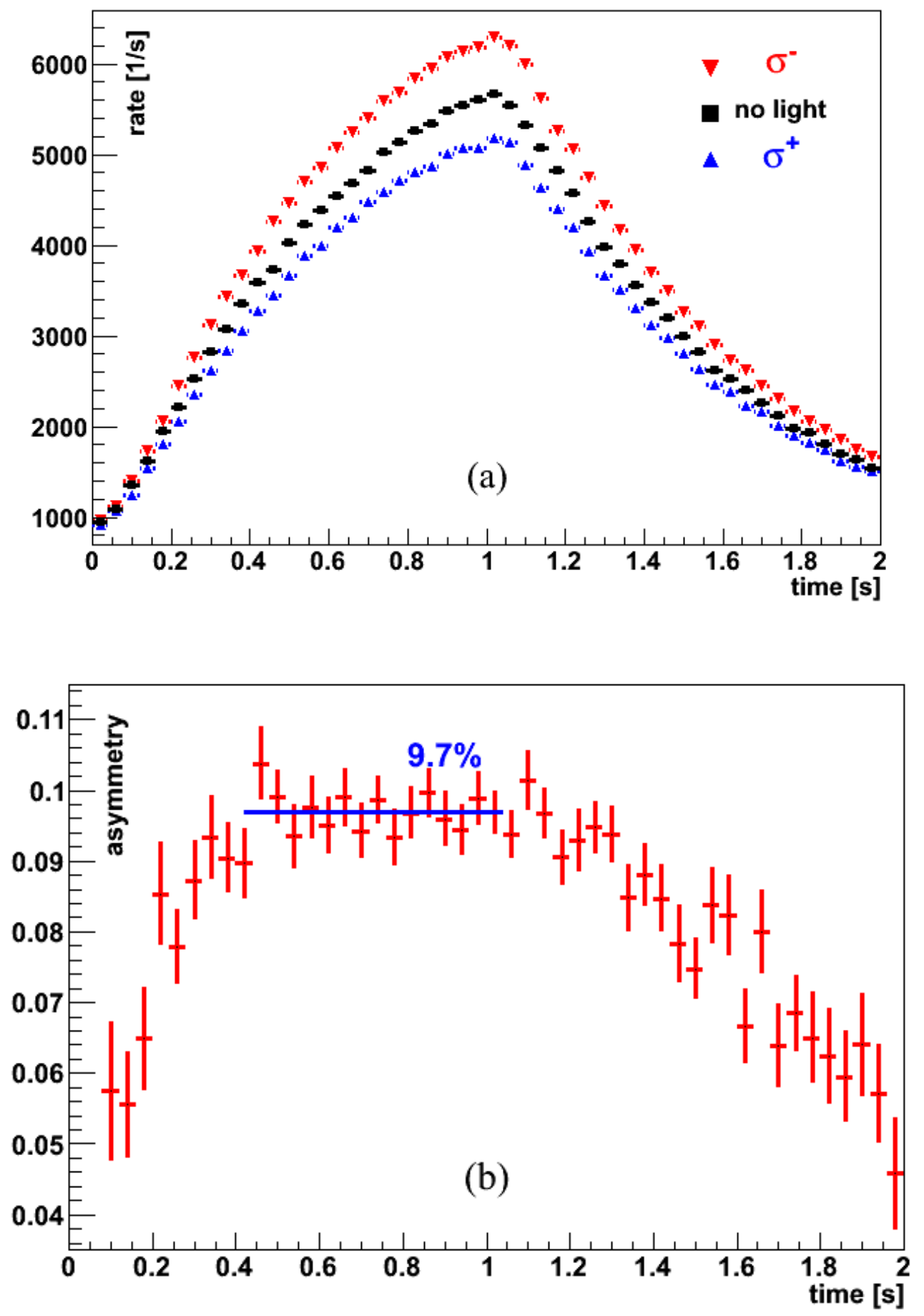

FIG. 3: (a) Rates detected with the $\beta$-detector for different helicity states of the laser light with a pulsed beam of ${ }^{20} \mathrm{Na}(0-1 \mathrm{~s}$ "on", 1 - $2 \mathrm{~s}$ "off"). Rates have been averaged over a data taking period of 35 min. (b) Corresponding asymmetry $\mathcal{A}_{\beta}$ from Eq. (3). Note the offset vertical scales. 
of the ${ }^{20} \mathrm{Na}$ atoms out of the laser light and molecule formation of sodium with residual chemically active contaminants in the buffer gas. This is consistent with the decreasing $\beta$ asymmetry when the ${ }^{20} \mathrm{Na}$ beam is switched off, as seen in Fig. 3b. When the dispenser with natural $\mathrm{Na}$ is heated, the polarization is also lower, due to convection in the buffer gas and/or additional unwanted materials that are heated off surfaces. After turning off the dispenser the polarization in the beam- "on" cycles maximizes and then slowly drops in the course of hours. We also note that not all stopped $\mathrm{Na}$ ions neutralize due to the ionizationenergy difference of $\mathrm{Na}$ and $\mathrm{Ne}$, cf. Ref. [14]. For these reasons we make the simplifying assumption that at the end of the production cycle $30 \%$ of the ${ }^{20} \mathrm{Na}$ is fully polarized while the remaining fraction is not.

The $\gamma$-ray rates, $R_{\gamma}^{\uparrow \downarrow}$, for the two polarization directions are used to determine the lifetimes $\tau^{\uparrow(\downarrow)}$ from which the LIV $\gamma$-ray asymmetry, $\mathcal{A}_{\gamma}$, is then constructed as

$$
\mathcal{A}_{\gamma}=\frac{\tau^{\uparrow}-\tau^{\downarrow}}{\tau^{\uparrow}+\tau^{\downarrow}}=P \xi_{2} \hat{n}_{2} \cdot \hat{z},
$$

where $\hat{n}_{2} \cdot \hat{z}$ is given in Eq. (2).

The $\gamma$-count-rate spectra of the 2-second cycles are combined as in Fig. 3a, accumulated in half-hour bins into three spectra: one for the unpolarized case and one for each of the two polarization directions. A count rate dependence on the gain of the photomultiplier tube could not be avoided, making the energy threshold of the trigger count rate dependent. In addition, pile-up occurs. Both dependencies require correction that is quadratic in the rate. The corresponding factor is determined using the non-polarized data set. After this correction, consistent fits with a single effective lifetime were obtained. The two $\gamma$ detectors have different responses and are analyzed separately; in the following the data are shown for one detector.

A residual dependence on count rate still remained (see Fig. 4). The average half-life for the nonpolarized data deviates $12 \mathrm{~ms}$ from the literature value $(447.9 \pm 2.3 \mathrm{~ms}$ [11]), while the results between the two polarization conditions differ by $3.1 \mathrm{~ms}$. Indeed, in Fig. 4 we see systematic variations in the fitted lifetimes occurring for all three modes simultaneously. This systematic error has serious consequences for the $\gamma$-ray asymmetry. The $\gamma$ detectors are not blind to positron emission; a fraction of the $\beta$ particles enter the detectors directly and, in addition, positrons that annihilate in the surrounding matter deposit radiation. Although the amount of energy can be too low to cross the threshold, coincident summing 


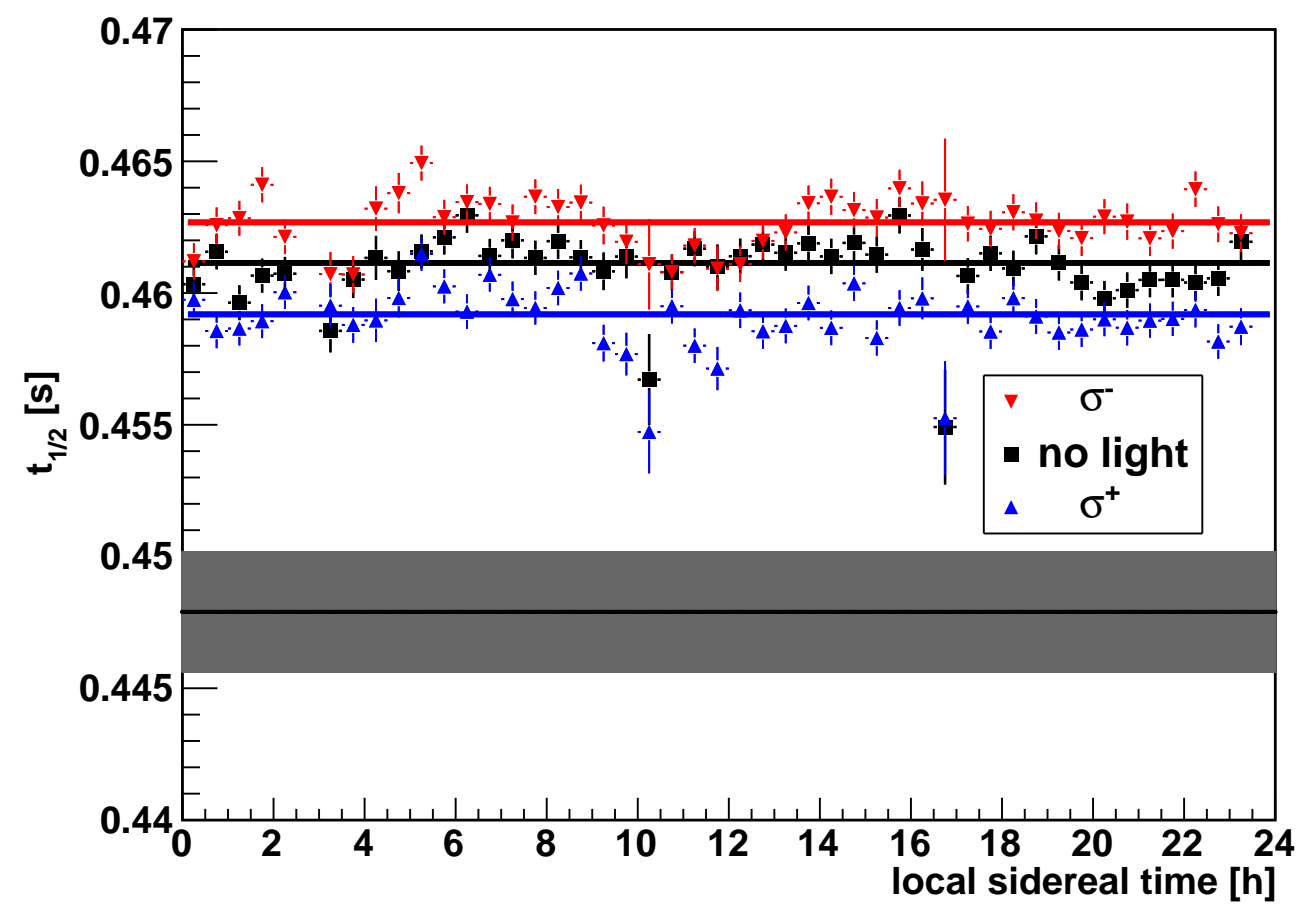

FIG. 4: Extracted lifetimes as a function of local sidereal time for the three polarization states (up, off, down). The accepted value for the lifetime of ${ }^{20} \mathrm{Na}$ is indicated by the dark band.

with incompletely detected $1.6 \mathrm{MeV}$ photons produces additional triggers. Because the polarization is time dependent this results in a changing count rate that makes the lifetime dependent on polarization. The effect is dependent on the polarization direction because the polarization axis is not exactly perpendicular to the axis of the $\gamma$ detectors, the detectors have different efficiencies, and the matter surrounding these detectors is not symmetric. However, the production and decay cycles are so short that the conditions within a few cycles are very similar and do not accumulate into differences on the sidereal time scale. Dividing the data without polarization in two sets separated by 6 seconds, an asymmetry constructed from these two sets as in Eq. (4) shows no sidereal dependence within the statistical accuracy. The setup is thus well suited to search for the sidereal dependence. For limiting the constant term of Eq. (2) it is not suited.

In Fig. 5 we show the lifetime asymmetry of Eq. (4). The offset in the data has been explained above. The sidereal time dependence obtained from a fit to the data does not deviate statistically significant from zero. Combining the data of both detectors gives a 


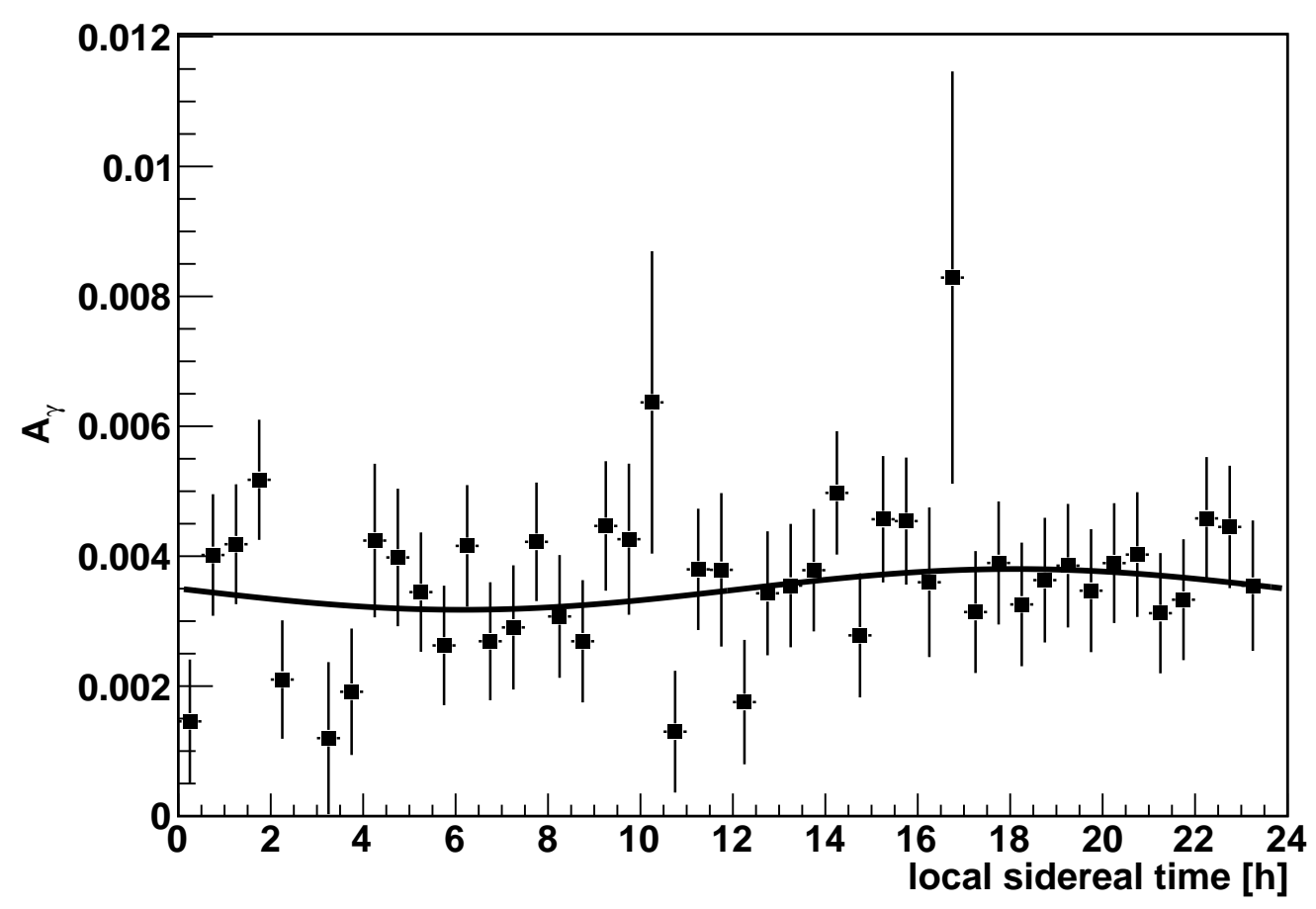

FIG. 5: The $\gamma$-ray asymmetry determined from the half-life differences between up and down polarization. The origin of the graph has been chosen to coincide with local sidereal time zero, to allow separate limits for the $N^{1,2}$ directions.

bound of $\left|\mathcal{A}_{\gamma}\right|<4 \times 10^{-4}$ at $95 \%$ confidence. The average polarization is $23 \%$ with an uncertainty due to the averaging procedure over the polarization lifetime of about $5 \%$. The colatitude of our institute is $37^{\circ}$. Therefore, the anisotropy in the equatorial plane is bound by $\left|\xi_{2}\right|<3 \times 10^{-3}$ at $95 \%$ C.L. In the top half of Table I the result is given for the two equatorial directions separately.

In the theoretical work [1] it was shown that a rather general extension of the standard model is obtained by adding a LIV tensor $\chi^{\mu \nu}$ to the W-boson propagator. For a GamowTeller transition the value

$$
\xi_{2} \hat{n}^{l}=A \epsilon^{l m k} \chi_{i}^{m k}=A \tilde{\chi}_{i}^{l}
$$

was found. Here $A$ is the $\beta$-asymmetry parameter, the index $i$ refers to the imaginary part of $\chi^{\mu \nu}$, while the indices $(k, l, m)$ refer to the spacelike components of $\chi^{\mu \nu}$. Transformed to the Sun-centered frame $\chi^{k l} \rightarrow X^{k l}$, which can be written in terms of standard model extension (SME) parameters [2]. $\left|\tilde{X}_{i}^{l}\right|$ depends on the antisymmetric part of $k_{\phi \phi}$ and on 
TABLE I: Limits on $\xi_{2} N^{1,2}$ and the corresponding SME parameters in the Sun-centered frame.

\begin{tabular}{|c|c|c|}
\hline \hline Coefficient & \multicolumn{1}{|c|}{ Value } & $95 \%$ C.L. interval \\
\hline$\xi_{2} N^{1}$ & $(-12 \pm 9) \times 10^{-4}$ & {$[-29,+6] \times 10^{-4}$} \\
$\xi_{2} N^{2}$ & $(-3 \pm 8) \times 10^{-4}$ & {$[-19,+14] \times 10^{-4}$} \\
\hline$\tilde{X}_{i}^{1}$ & $2\left(k_{\phi \phi}^{A}\right)^{32}+\frac{1}{g}\left(k_{\phi W}\right)^{32}$ & {$[-9,+2] \times 10^{-3}$} \\
$\tilde{X}_{i}^{2}$ & $2\left(k_{\phi \phi}^{A}\right)^{13}+\frac{1}{g}\left(k_{\phi W}\right)^{13}$ & {$[-6,+4] \times 10^{-3}$} \\
\hline \hline
\end{tabular}

$k_{\phi W}$ [1]. The corresponding expressions and the limits at 95\% C.L. are given in the bottom half of Table I. A value for $\tilde{X}_{i}^{3}$ cannot be obtained with the present setup. In Ref. [1] it is shown how the real part of $\chi^{\mu \nu}$ can be obtained in different $\beta$-decay measurements exploiting also the different dependence of Fermi and Gamow-Teller transitions on $\chi^{\mu \nu}$.

A setup optimized for $\tilde{X}_{i}^{1}$ and $\tilde{X}_{i}^{2}$ should have its polarization perpendicular to the Earth's rotation axis. Such a measurement, where, in addition, the $\gamma$ detection is much less sensitive to $\beta$ particles is currently being analyzed.

In summary, $\beta$ decay can be exploited to search for Lorentz invariance violation in the weak interaction, which has not been searched for yet in any systematic way. The framework developed in Ref. [1] makes this possible by parametrizing the LIV in terms of a background tensor $\chi^{\mu \nu}$. The current work is the first attempt to put limits on the magnitude of $\chi^{\mu \nu}$. A limit was set on the imaginary part of the spacelike component of $\chi^{\mu \nu}$ by searching for a variation in the decay rate of ${ }^{20} \mathrm{Na}$ nuclei as function of their spin direction. A limit of a sidereal dependence in the equatorial plane of $3 \times 10^{-3}$ at $95 \%$ C.L. was found, independent of theory. The theoretical framework implies that with this experiment and a variety of other experiments one can limit the magnitude of the LIV tensor further.

We thank the KVI cyclotron staff for providing the beam and L. Huisman for technical support. This research was financially supported by the "Stichting voor Fundamenteel Onderzoek der Materie (FOM)" under Program 114 (TRI $\mu \mathrm{P})$ and "FOM projectruimte" 08PR2636-1. 
* Present address: Institute of Radiation Physics, Helmholtz-Zentrum Dresden-Rossendorf, Germany.

$\dagger$ Present address: Max Planck Institute for Nuclear Physics, Heidelberg, Germany.

$\ddagger$ Electronic address: wilschut@kvi.nl

$\S$ University of Osaka, Osaka, Japan

[1] J.P. Noordmans, H.W. Wilschut, and R.G.E. Timmermans, Phys. Rev. C 87, 055502 (2013).

[2] D. Colladay and V.A. Kostelecky, Phys. Rev. D 58, 116002 (1998).

[3] G. Amelino-Camelia et al., Eur. Phys. J. C 68, 619 (2010); V.A. Kostelecky and R.J. Van Kooten, Phys. Rev. D 82, 101702(R) (2010).

[4] V.A. Kostelecky, Phys. Rev. D 64, 076001 (2001), and references therein.

[5] J.S. Diaz, V.A. Kostelecky, R. Lehnert, Phys. Rev. D. 88, 071902 (2013).

[6] J.P. Noordmans, H.W. Wilschut, and R.G.E. Timmermans, Phys. Rev. Lett. 111, 171601 (2013).

[7] H.W. Wilschut et al., Ann. Phys. (Berlin) 525, 653 (2013).

[8] R. Newman and S. Wiesner, Phys. Rev. D 14, 1 (1976).

[9] J. D. Ullman, Phys. Rev. D 17, 1750 (1978).

[10] V. A. Kostelecky and N. Russell, Rev. Mod. Phys. 83, 11 (2011).

[11] D.R. Tilley, C.M. Cheves, J.H. Kelley, S. Raman, and H.R. Weller, Nucl. Phys. A636, 249 (1998).

[12] E. Traykov et al., Nucl. Instrum. Methods Phys. Res., Sect. A 572, 580 (2007).

[13] E.A. Dijck, Master's Thesis, University of Groningen, 2012, http://irs.ub.rug.nl/dbi/50486994be7e0.

[14] P. Dendooven, S. Purushothaman, and K. Gloos, Nucl. Instrum. Methods Phys. Res., Sect. A 558, $580(2006)$. 